\title{
Le contre-cycle économique se poursuit au Saguenay-Lac-St-Jean (Radioscopie comparative : Larouche vs Proulx)
}

\author{
Sylvain Larouche \\ Université du Québec à Chicoutimi
}

\section{INTRODUCTION}

Malgré que la région du S-L-S-J avait su prendre le contre-cycle économique technologique et crôitre au même rythme que la moyenne québécoise, ce virage masquait une restructuration d'un nouveau cycle de développement régional qui ne semblait pas être au rendez-vous.

En 2007, Proulx ${ }^{1}$ écrivait le livre «Le Saguenay-Lac-Saint-Jean face à son avenir $»^{2}$. Dans le chapitre 3, l'auteur faisait l'état d'un contre-cycle économique important ${ }^{3}$ qui frappait la région du Saguenay-Lac-Saint-Jean (S-L-S-J) depuis le début des années 1980. Malgré que la région du S-L-S-J avait su prendre le contrecycle économique technologique et croître au même rythme que la moyenne québécoise, ce virage masquait une restructuration d'un nouveau cycle de développement régional qui ne semblait pas être au rendez-vous. Des ménages appauvris, une certaine rareté des activités postindustrielles, un entreprenariat faible, des innovations en entreprise peu nombreuses et les replis corporatifs de plus en plus légion étaient là pour confirmer que la planification régionale globale était partielle, conservatrice et visiblement peu innovatrice.
Selon cette étude, l'auteur affirme « qu'en définitive, la collectivité territoriale du S-L-S-J était enlisée dans un contre cycle structurel, incapable d'accélérer le pas vers le nouveau cycle économique. Les pertes d'emplois industriels et les difficultés dans le secteur forestier n'auguraient rien de bon pour cette économie régionale qui semblait incapable de s'insérer convenablement dans la mutation rapide vécue à l'échelle mondiale $»^{2}$. Ce diagnostic semblait dur à l'endroit de la situation du S-L-S-J en 2007, mais tous les indicateurs économiques de l'époque appuyaient et confirmaient le fait que la région s'enlisait dans un contre-cycle structurel.

Maintenant en 2012, qu'en est-il de ce contrecycle? Malgré plusieurs avertissements, est-ce que la région du S-L-S-J a su mettre en place les actions structurantes nécessaires à son redressement? Est-ce que les intervenants stratégiques de la région ont fait les efforts nécessaires pour adopter des scénarios de relance du cycle structurel? Ce sont là les questions qui serviront d'étalonnage pour mesurer l'évolution de la situation au cours des cinq dernières années. À cette fin, une radioscopie actualisée de l'environnement socio-économique de la région du Saguenay-Lac-Saint-Jean est nécessaire.

\section{RADIOSCOPIE RÉGIONALE ACTUELLE EN 2012}

\section{La démographie}

Même si la situation demeure précaire et que les perspectives demeurent négatives il est tout de même intéressant de constater que la baisse démographique s'est arrêtée en 2011.

La situation démographique du S-L-S-J est inquiétante, puisque depuis les dernières décennies la population ne cesse de diminuer. Selon le portrait régional rédigé à l'été 2012 sur la situation du S-L-S-J, par le gouvernement du Québec ${ }^{4}$, de 2006 à 2011, la population a continué de décroitre de $0,2 \%$ alors qu'à l'inverse, le Québec enregistrait une croissance de 4,6\%. La région du S-L-S-J représente $3,43 \%$ de la population en 2011 alors qu'en 2006 elle représentait $5 \%$ de la population globale du Québec avec ses 273000 
habitants 5 . Les perspectives vont dans le même sens qu'en 2007, soit une décroissance de l'ordre de $1,2 \%$ annuellement. Les mouvements migratoires, responsables de nombreux déclins démographiques observés dans les régions, sont également déficitaires depuis 2006 (500 personnes par années). Cependant, en 2011, trois personnes de plus que ceux qui ont quittés viennent confirmer un premier gain depuis plusieurs années. Selon une étude régionale de Desjardins basée sur l'Institut de la statistique du Québec ${ }^{4}$, la population totale en 2011 était de 273461 soit 461 résidants de plus qu'en 2007. Même si la situation demeure précaire et que les perspectives demeurent négatives il est tout de même intéressant de constater que la baisse démographique s'est arrêtée en 2011. Cependant notre poids démographique avec le reste du Québec c'est, quant à lui, résorbé de $0,5 \%$.

\section{PRINCIPAUX INDICATEURS ÉCONOMIQUES}

\section{L'emploi}

\section{Sur le plan de l'emploi, pour la région du S-L-S-J, la situation s'améliore et semble prendre une tendance prometteuse.}

Selon l'institut de la statistique du Québec ${ }^{6}$, le taux de chômage au S-L-S-J s'établissait à 7,5\% en juillet 2012 comparativement à $8,3 \%$ en 2011 et $8,1 \%$ en 2010. Il était de $13 \%$ en 2007. Il est inférieur de $0,2 \%$ par rapport au taux de chômage observé dans le reste du Québec qui se situe à 7,7\%. Le taux de chômage saguenéen a baissé de près de $1 \%(0,8 \%)$ par rapport à l'année précédente alors que celui de la province de Québec toute entière n'a diminué que de $0,1 \%$. Fait intéressant à noter, en milieu d'année 2012, par rapport à 2011, la région avait 300 emplois de plus. En bref, sur le plan de l'emploi, pour la région du S-L-S-J, la situation s'améliore et semble prendre une tendance prometteuse.

\section{Investissements}

Il y eu entre 2008 et 2012 au S-L-S-J, une augmentation annuelle moyenne des investissements de l'ordre de $9 \%$.

Concernant les investissements, en 2007, c'était ceux du secteur privé qui permettait à la région de se maintenir par rapport à l'ensemble du Québec. En 2012, il en va tout autrement. Selon l'Institut de la statistique du Québec ${ }^{7}$, la province de Québec aura une croissance de ses investissements de l'ordre de 5,6\% alors qu'au S-L-S-J, suivant une progression de 35,5\% entre 2010 et 2011 , cette croissance sera réduite de
$10 \%$. Le S-L-S-J sera premier pour les investissements dans les industries productrices de services $(17,2 \%)$ mais bon dernier dans les investissements du secteur privé non résidentiel avec un $-26,1 \%$. Provenant essentiellement des gouvernements (Plan nord, etc.), les investissements publics seront de $20 \%$, soit les plus importants au Québec. Mentionnons en terminant que malgré une diminution des investissements par rapport à 2011, il y eu entre 2008 et 2012 au S-L-S-J, une augmentation annuelle moyenne des investissements de l'ordre de $9 \%$.

\section{Production}

Selon Desjardins, en 2011 le PIB nominal a augmenté de 4,7\% par rapport à 2010, mais de seulement 5,6\% comparé à 2007. Dans l'ensemble des régions administratives du Québec, la variation s'est également chiffrée à 4,7 pour les années 2010-2011, mais de $13 \%$ si on le compare avec l'année 2007. Par habitant, l'écart entre 2010 et 2011 est sensiblement identique au PIB nominal soit $4,5 \%$. A priori, ce résultat semble intéressant puisque l'augmentation annuelle du PIB par habitant au S-L-S-J est de 0,2 point supérieur à celui du reste du Québec $(4,3 \%)$. Toutefois, lorsqu'on le compare avec 2007, on dénote un écart important puisque celui du Québec en entier a augmenté de $9,39 \%$ relativement à celui du S-L-S-J qui a été de 5,37\%.

\section{Revenus par habitant}

Toujours selon Desjardins, en 2011 le revenu personnel disponible par habitant a progressé de $3,8 \%$ au S-L-S-J comparativement à 3,6 \% pour les autres régions administratives du Québec. Malgré cette différence et le fait qu'entre 2007 et 2011 nous avons observé une augmentation plus 
marqué du revenu personnel disponible par habitant au S-L-S-J (15\% versus $12 \%)$, il continue d'y avoir un gap de $1856 \$$ par habitant à l'avantage des Québécois demeurant hors de la région du S-L-S-J.

\section{Faillites}

Le nombre de faillites commerciales et personnelles ne cesse de diminuer depuis 2007 au

\section{DÉVELOPPEMENT RÉGIONAL}

\section{La désappropriation du secteur privé}

S'inspirant de l'analyse économique du ministère Développement Économique, Innovation et Exportation (MDEIE) aujourd'hui ministère des Finances et du Développement sur le renouvellement de l'entrepreneuriat au Québec incluant le S-L-S-J ${ }^{10}$, il y avait 180800 entrepreneurs actifs au Québec en 2008. Il y en aura 5000 de moins en 2013. Une variation négative de $2,8 \%$. Si la tendance se maintient, on estime qu'entre 2008 et 2018, cette variation négative passera à $13,9 \%$. Cet écart entre le nombre d'éclosions et de retraits se traduira par une diminution de 25200 du nombre d'entrepreneurs depuis 2008 . Cette situation aura des impacts négatifs au Québec et, par le fait même, au S-L-S-J, car il sera ardu de soutenir la création d'emploi, d'alléger la pauvreté et de renouveler la structure industrielle par l'innovation.

\section{Le secteur de l'aluminium}

Depuis la crise de 2009, le marché mondial de l'aluminium se redresse. Au S-L-S-J, les perspectives font état d'une éventuelle croissance de l'ordre de $2,3 \%$ du secteur de la fabrication de 2011 à 2012 comparativement à $0,5 \%$ pour l'ensemble du Québec ${ }^{8}$. Ce secteur représente $13,3 \%$ de l'ensemble de l'emploi régional. Cette croissance aura un impact significatif sur l'emploi qui devrait croître de $2,4 \%$ pour les trois prochaines années pour le S-L-S-J. Malgré cela, la relance est prudente. Il est possible qu'il y ait 3 facteurs qui expliquent cette timidité : d'abord, toutes les alumineries veulent gérer l'offre du métal efficacement de façon à ce que les prix remontent. Ensuite, les négociations entre les États nationaux et les producteurs d'aluminium sont serrées, car chacun désire améliorer ses
S-L-S-J ${ }^{6}$. Dans le Québec tout entier les faillites commerciales et personnelles ont régressées, mais nullement à la hauteur de celles du S-L-S-J. En 2010, par rapport à l'année précédente, les faillites personnelles ont chutées de $40 \%$ au S-L-S-J versus $32 \%$ pour le Québec, alors qu'elles ont dégringolés de $53 \%$ versus $37 \%$ dans le secteur commercial.

conditions économiques. Enfin, les grandes alumineries se parlent de manière à contrôler la production mondiale. Cependant, cette situation achève. Appuyé par une demande mondiale en croissance, le prix de la tonne d'aluminium devrait se situer entre 3200 et 3400 \$US d'ici quelques années, ce qui est de bon augure, puisque le niveau d'équilibre oscille entre 2300 et 2500 \$US. En septembre 2012, le prix de l'aluminium se situe autour de 2190 \$ US la tonne. Au niveau des investissements, la région du S-L-S-J devrait bien s'en sortir puisque Rio Tinto Alcan devrait débuter les phases 2 et 3 du projet AP60 en 2012-2013 avec des coûts d'investissement estimés à $3,7 \mathrm{G} \$$.

\section{L'Hydroélectricité}

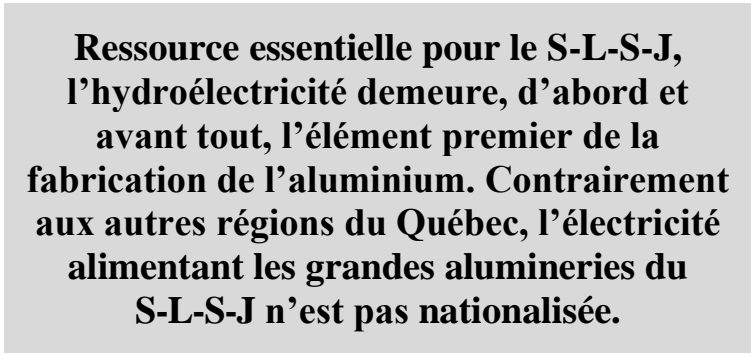

Ressource essentielle pour le S-L-S-J, l'hydroélectricité demeure, d'abord et avant tout, l'élément premier de la fabrication de l'aluminium. Contrairement aux autres régions du Québec, l'électricité alimentant les grandes alumineries du S-L-S-J n'est pas nationalisée. Cela procure un avantage concurrentiel certain à Rio Tinto Alcan. Selon Proulx « Le réel potentiel théorique futur de 1'hydrographie québécoise offre la possibilité de doubler la production hydroélectrique actuelle ${ }^{9}$. Cependant, il mentionne également que dans la même foulée $50 \%$ de 
l'hydroélectricité produite en Amérique du Nord est vendue à l'industrie pour stimuler l'économie et la création d'emploi. Aussi, pour toutes les raisons évoquées précédemment, rien de bien important dans ce secteur, n'étant pas relié à l'industrie de l'aluminium, ne devrait émerger au S-L-S-J dans les prochaines années.

$50 \%$ de l'hydroélectricité produite en

Amérique du Nord, est vendue à l'industrie pour stimuler l'économie et la création d'emploi.

\section{La filière forêt}

Dans le secteur de l'industrie forestière, le taux de croissance annuel moyen de l'emploi entre 2011-2013 devrait augmenter de 1,5\% comparativement à $0,9 \%$ pour le reste du Québec ${ }^{8}$. Ce scénario repose sur la reprise de la construction aux États-Unis, sur les campagnes de sensibilisation pour l'utilisation du bois dans l'industrie de la construction et sur les produits forestiers écologiques ou la région du S-L-S-J a déjà deux unités d'aménagement certifiées (norme FSC). En contrepartie, selon le rapport sectoriel de Service Canada, l'emploi devrait diminuer de $9 \%$ annuellement pour la période 2011-2012. Fusions et fermetures seront la conséquence d'une recherche d'une meilleure productivité par les entreprises de plus en plus axées sur les technologies de l'information.

\section{Le secteur minier}

Grâce au secteur minier, la région du S-L-S-J profitera d'une augmentation significative de ses investissements. Les investissements pourraient être encore plus élevés dans la mesure où les trois projets miniers d'exploitation et de développement actuellement en phase d'analyse et de faisabilité se réaliseraient, soit l'exploitation d'une mine de phosphate-titane au Lac à Paul $(350 \mathrm{M})$, le développement d'une mine de niobium et de tantale au nord de Saint-Félicien $(315 \mathrm{M})$ et celui des mines de diamants et d'uranium des monts Otish $(802 \mathrm{M})^{5}$.

\section{L'agriculture}

Malgré l'arrivée de l'agriculture nordique (bleuets sauvages, pommes de terre de semence, petits fruits, canneberges, etc.) qui s'avère très populaire auprès des consommateurs, l'industrie agricole devrait décroître à un rythme annuel moyen de $1 \%$ selon Service Canada. En effet, la baisse importante de fermes se poursuivra et le nombre d'exploitants continuera de reculer. Les économies d'échelle provoquées par la mondialisation viendront décourager la relève qui se fait déjà de moins en moins présente.

\section{Le tertiaire moteur}

S'appuyant sur le bulletin régional de juillet 2012 de 1'Institut de la statistique du Québec ${ }^{11}$, on constate que le secteur tertiaire continu d'être un élément moteur pour le développement économique de la région du S-L-S-J. En fait, lorsqu'on compare l'année 2011 avec l'année 2007, le nombre d'emplois dans le secteur tertiaire est demeuré stable; $73,6 \%$ de la totalité des emplois en 2011 versus 72,39\% en 2007. Cependant, il est important de signaler qu'entre 2007 et 2011, il s'est perdu 3300 emplois dans ce secteur soit approximativement le même nombre que dans le secteur des biens.

\section{Le secteur tertiaire continu d'être un élément moteur pour le développement économique de la région du Saguenay-Lac- St-Jean.}

\section{Le tourisme}

En 2011, selon l'étude économique du mouvement Desjardins, , le taux d'occupation hôtelier en 2011 était inférieur de $0,5 \%$ en comparaison avec 2007, soit $38,4 \%$ versus $38,9 \%$. Le taux de croissance de cette industrie demeure stable malgré tous les efforts que la région du S-L-S-J a fait pour revigorer ce secteur. Depuis les cinq dernières années, le taux d'occupation au S-L-S-J demeure à $13 \%$ en dessous de la moyenne nationale $(51,3 \%)$.

\section{Le plan nord}

Selon l'étude économique de Desjardins ${ }^{5}$, dans la marche pour devenir la plaque tournante pour le développement du Nord, la région a déjà investi $10 \mathrm{M}$ dans la création d'un parc industriel à l'aérogare de Bagotville et à la construction d'un port en eau profonde à Grande-Anse, relié par voie ferrée. Combiné aux infrastructures en 
place, au parachèvement de la route 175 ainsi qu'à l'expertise régionale, la région du S-L-S-J a de nombreux atouts pour parvenir à ses fins.

\section{L'éolien}

L'énergie éolienne sera développée au S-L-S-J avec un projet de construire le plus important parc éolien au Canada ( $800 \mathrm{M}$ ) dans la MRC du Fjorddu-Saguenay ainsi que celle de Charlevoix ${ }^{5}$.

\section{ANALYSE COMPARATIVE}

À la lueur des informations recueillies sur les cinq dernières années (2008 à 2012) on constate que le contre-cycle structurel se poursuit. Dans le tableau 1 les conclusions de la radioscopie de Proulx sur certains éléments clés en 2007 versus la radioscopie actualisée de 2012.

\section{Étant au dernier rang en matière} d'investissements privés au S-L-S-J en 2012, un signal sérieux s'ajoute : un effritement de l'entrepreneurship qui se poursuit, une faiblesse de l'innovation qui demeure et une désappropriation du secteur privé qui s'intensifie.

Même si le tableau 1 n'a aucune prétention et pondération, il met tout de même certains faits en évidences. D'un premier coup d'œil, on constate qu'il n'y a pas eu de régression au S-L-S-J. La filière forêt inquiète, mais les secteurs de l'aluminium et de l'éolien rassurent. Même constat pour le secteur de la production qui inquiète, mais heureusement, qui se fait plus discret grâce à la performance de l'emploi. Les faillites sont à la baisse, mais le nombre d'entrepreneurs ne cesse de diminuer. Les autres indicateurs économiques et sociaux (investissements, revenus, hydroélectricité, tourisme, agroalimentaire) bien que différents quelquefois dans leur finalité d'analyse, semblent fixes, ou bien sans grandes distinctions avec l'année 2007. La démographie, bien que stable en 2011, alarme encore avec des baisses prévues pour les prochaines années de l'ordre de 1,2\% annuellement. Étant au dernier rang en matière d'investissements privés au S-L-S-J en 2012, un signal sérieux s'ajoute: un effritement de l'entrepreneurship qui se poursuit, une faiblesse de l'innovation qui demeure et une désappropriation du secteur privé qui s'intensifie. Enfin, le secteur

\section{Science et technologie}

En 2010, le niveau de l'emploi des personnes de 25 à 64 ans occupées en science et technologie dans la région était pratiquement le même qu'en 2006 (101,9 contre 112,7 au Québec). Selon Emploi-Québec, d'ici 2012, il y aura 5000 emplois à pourvoir dans ce domaine dans la région ${ }^{5}$.

tertiaire au S-L-S-J en 2012 (17,2 \% d'investissement dans ce secteur), bien que toujours moteur, n'est pas aussi dynamique qu'anticipé.

Même si la situation semble stable, le contrecycle économique au S-L-S-J n'est pas terminé. Malgré une vision commune au début des années 2000 axée sur l'innovation et l'introduction de changements constants, la région du S-L-S-J est demeurée dans un modèle de développement purement fonctionnel. Les cinq vocations de l'oasis nordique telles qu'exposées dans le projet «Vision $2025 »^{2}$, bien qu'ambitieuses, étaient réalisables et pourtant le contrecycle s'est poursuivi. Le S-L-S-J s'était doté d'une planification territoriale avant-gardiste mais lorsque le moment fut venu d'agir, l'effet d'interaction escompté n'a pas été suffisant et les principales recommandations d'alors n'ont pas été suivies avec diligence.

Malgré cela, le projet «Vision 2025 » demeure d'actualité pour sortir durablement la région $\mathrm{du}$ S-L-S-J de ce contre-cycle structurel. En fait, il demeure la référence et les intervenants régionaux devront se mobiliser en fonction d'objectifs communs axés sur l'émergence d'une communauté apprenante prospère, une communauté de l'an 2025.

Même si la situation semble stable, le contre-cycle économique au S-L-S-J n'est pas terminé. Malgré une vision commune au début des années 2000 axée sur l'innovation et l'introduction de changements constants, la région du S-L-S$J$ est demeurée dans un modèle de développement purement fonctionnel. 


\section{Tableau 1 - Radioscopie actualisée 2012 vs 2007}

\section{7 \\ (Radioscopie Marc-Urbain Proulx) La démographie (Amélioration) \\ La démographie régionale est décroissante depuis 1981 et ce déclin s'est dramatiquement accéléré depuis 1996}

Malgré ses pertes d'emplois massives la région métropolitaine de Saguenay demeure en bonne position par rapport à sa région du S-L-S-J.

\section{Investissements (Stable)}

Ce sont les investissements majeurs effectués par le secteur privé qui permettent à la région sa part relative par rapport à l'ensemble du Québec.

\section{Production (Abaissement)}

Les données disponibles sur la production régionale attestent d'une performance relative de la région du S-L-S-J supérieure à la moyenne québécoise.

\section{L'emploi (Amélioration)}

2012

(Radioscopie actualisée)

Même si la situation demeure précaire et que les perspectives demeurent négatives il est tout de même intéressant de constater que la baisse démographique s'est arrêtée en 2011. Cependant notre poids démographique avec le reste du Québec c'est, quant à lui, résorbé de $0,5 \%$.

\section{Revenus (Stable)}

Si le revenu moyen disponible pour les travailleurs québécois tend à diminuer légèrement en dollars constants entre 1973 et 2002, force est de constater que ce phénomène d'appauvrissement réel est encore plus présent au S-L-S-J.

\section{Faillites (Amélioration)}

La situation économique de la région se détériore davantage sous cet indicateur, malgré une conjoncture économique favorable actuellement sous l'angle de la production.

\section{Désappropriation du secteur privé (Abaissement)}

Le mouvement lourd d'érosion de la propriété locale et régionale des activités économiques a des effets négatifs considérables sur l'économie régionale, notammen en participant à la réduction de son autosuffisance et de sa capacité d'impulsion endogène de son développement.

\section{Aluminium (Amélioration)}

Si la production primaire d'aluminium est concurrentielle dans la région, la structuration de la filière industrielle ne s'effectue que très lentement malgré les efforts publics et privés.

\section{Hydroélectricité (Stable)}

Les projets hydroélectriques en cours et envisagés dans la périphérie nordique nécessitent un prise de position claire du S-L-S-J à l'égard de la dualité du marché potentiel.

\section{Forêt (Abaissement)}

Le secteur de la forêt fait face à des défis importants dans la région. Pour les relever adéquatement les experts et les acteurs devront innover sous de nombreux aspects vitaux de cette indus

\section{Agroalimentaire (Stable)}

En évolution rapide et constante l'agroalimentaire subit de plein fouet le mouvement d'intégration des grandes unités de production qui échappent largement à la région dans leur choix de localisation optimale par rapport aux grands marchés. Parallèlement se multiplient avec bonheur et espoir les productions distinctives dans des créneaux.

\section{Le tertiaire (Stable)}

Malmené aussi par l'intégration sectorielle, notamment dans le génie-conseil, le secteur tertiaire moteur se porte assez bien et offre même des perspectives intéressantes pour le futur si les virages collectifs sont effectués vers les bons champs de spécialis

\section{Tourisme (Stable)}

Le S-L-S-J possède un potentiel récréotouristique considérable dans le contexte d'un secteur d'activité relativement stagnant dans lequel la promotion s'effectue d'une manière éclatée selon les divers intérêts

\section{Éolien (Amélioration)}

L'énergie éolienne offre pour le S-L-S-J un potentiel important de création de richess en raison d'une part, de la nouvelle compétitivité de cette source et, d'autre part, du flou qui caractérise actuellement ses conditions d'exploitation.
Selon I'Institut de la statistique du Québec, le taux de chômage au S-L-S-J s'établissait à 7,5\% en juillet 2012 comparativement à $8,3 \%$ en 2011 et $8,1 \%$ en 2010.

II $y$ eu entre 2008 et 2012 au S-L-S-J, une augmentation annuelle movenne des investissements de l'ordre de $9 \%$ faisant passer les investissements qui étaient alors de $1,8 \mathrm{M}$ à $2,5 \mathrm{M}$ en 2012 .

Toutefois, lorsqu'on le compare avec 2007, on dénote un écart important puisque celui du Québec en entier a augmenté de 9,39\% relativement à celui du S-L-S-J qui a été de $5,37 \%$. demeurants hors de la région du S-L-S-J.

Le nombre de faillite commerciale et personnelle ne cesse de diminuer depuis 2007 au S-L-S-J.

Il y avait 180800 entrepreneurs actifs au Québec en 2008. II y en aura 5000 de moins en 2013. Cette situation aura des impacts négatifs au Québec et, par le fait même, au S-L-S-J car il sera ardu de soutenir la création d'emploi, d'alléger la pauvreté et de renouveler la structure industrielle par l'innovation.

Le marché mondial de l'aluminium se redresse. Au S-L-S-J, les perspectives font état d'une éventuelle croissance de l'ordre de $2,3 \%$ du secteur de la fabrication de 2011 à 2012 comparativement à $0,5 \%$ pour l'ensemble du Québec.

Ressource essentielle pour le S-L-S-J, I'hydroélectricité demeure, d'abord et avant tout, l'élément premier de la fabrication de l'aluminium.

L'emploi diminuera de $9 \%$ annuellement pour la période 2011-2012. Fusions et fermetures seront la conséquence d'une recherche d'une meilleure productivité par les entreprises de plus en plus axées sur les technologies de l'information.

Malgré l'arrivée de l'agriculture nordique (bleuets sauvages, pommes de terre de semence, petits fruits, canneberges, etc.) qui s'avère très populaire auprès des consommateurs, l'industrie agricole devrait décroître à un rythme annuel moyen de $1 \%$

En fait, lorsqu'on compare l'année 2011 avec l'année 2007, le nombre d'emplois dans le secteur tertiaire est demeuré stable. $73,6 \%$ de la totalité des emplois en 2011 versus $72,39 \%$ en 2007 .

Le taux de croissance de cette industrie demeure stable malgré tous les efforts que la région du S-L-S-J a fait pour revigorer ce secteur. Depuis les cinq (5) dernières années, le taux d'occupation au S-L-S-J demeure $13 \%$ en dessous de la moyenne nationale $(51,3 \%)$

L'énergie éolienne sera développée au S-L-S-J avec un projet de construire le plus important parc éolien au Canada (800 M) dans la MRC du Fjord-du-Saguenay ainsi que celle de Charlevoix
Il continue d'y avoir un gap de 1856 \$ par habitant à l'avantage des québécois 


\section{BIBLIOGRAPHIE ET NOTES}

${ }^{1}$ Marc-Urbain Proulx est Docteur en économie régional et professeur à l'Université du Québec à Chicoutimi depuis 1988. Depuis septembre 2012, il est sousministre associé au ministère des Affaires municipales, des Régions et de l'Occupation du territoire et Directeur du centre de recherche sur le développement territorial (CRDT).

${ }^{2}$ Document référence décrit en début d'analyse de : Proulx, Marc-Urbain, 2007, « Le Saguenay-Lac-SaintJean face à son avenir », chapitre 3, Québec, Presses de l'université du Québec, pp. 35-102.

${ }^{3}$ Ses conclusions s'appuient sur des études fiables qui sont très bien identifiées dans le volume.

${ }^{4}$ Direction de l'analyse économique, gouvernement du Québec, MDEIE, Été 2012, Saguenay-Lac-Saint-Jean / Portrait régional, p.5.

${ }^{5}$ Desjardins - Études économiques, juin 2012, Survol de la situation économique, Région administrative du Saguenay-Lac-Saint-Jean, Volume 7 / Région 02, p.2.

${ }^{6}$ Institut de la statistique du Québec, Stat-EXPRESS, Août 2012, Bulletin hebdomadaire des indicateurs économiques conjoncturels, Vol. 14, n. 34.

${ }^{7}$ Institut de la statistique du Québec, Bulletin FLASH, 2012, Investissements privés et publics, Québec et ses régions,

${ }^{8}$ Lachance, Jean-Pierre, (2011). Perspectives sectorielles 2011-2012 - Saguenay-Lac-Saint-Jean, Service Canada, p.10.

${ }^{9}$ Proulx, Marc-Urbain, (2011). Territoires et développement : La richesse du Québec, Les presses de l'Université du Québec, p.11.

${ }^{10}$ Ministère développement économique, innovation et exportation (MDEIE), Le renouvellement de l'entrepreneuriat au Québec : un regard sur 2013 et 2018, publié par la Direction générale des communications et des services à la clientèle, 26 p.

${ }^{11}$ Bulletin statistique régionale, Édition 2012, $3{ }^{\mathrm{e}}$ trimestre, Institut de la statistique du Québec, Régions Saguenay-Lac-Saint-Jean, Bibliothèque et archive nationales du Québec, p.9.

${ }^{12}$ Proulx, Marc-Urbain, 24 mars 2010, Encore de la retenue dans l'aluminium, Journal «Le Quotidien», Saguenay-Lac-Saint-Jean. 


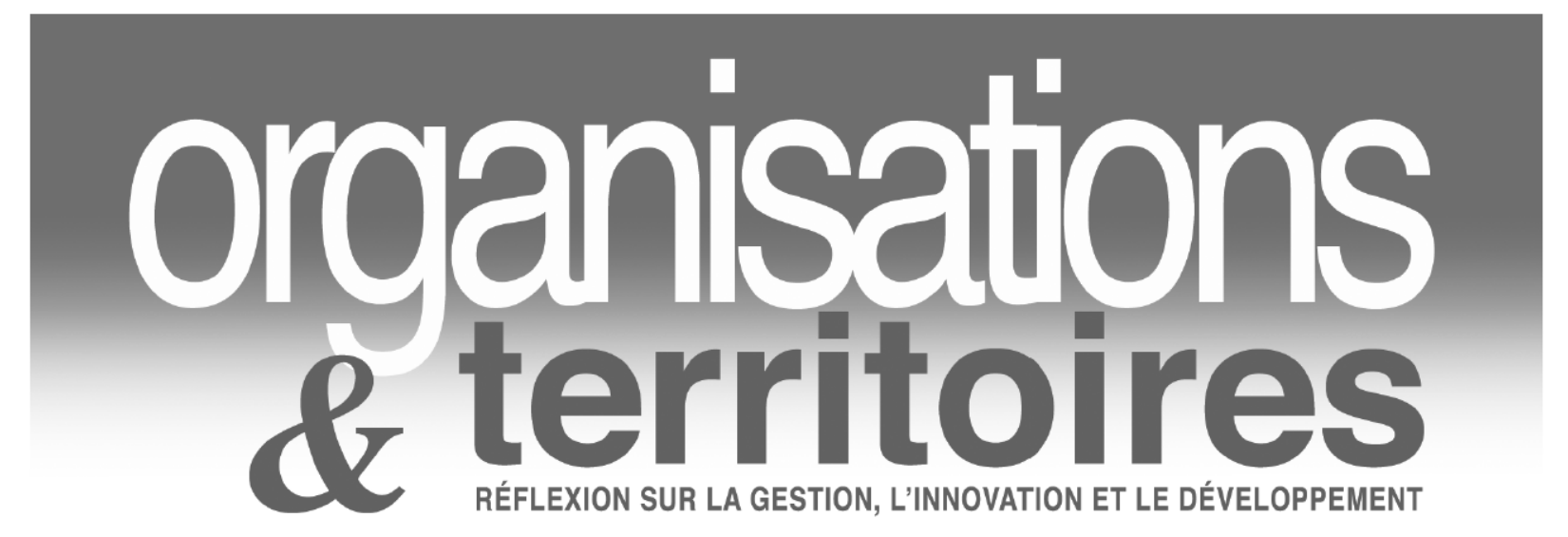

\section{Site Web}

\section{WWW.uqac.ca/revueot www.uqac.ca/revueot www.uqac.ca/revueot www.uqac.ca/revueot}

Nous vous invitons à l'explorer et à nous faire parvenir vos commentaires et suggestions afin d'en améliorer le contenu et la présentation. 